\title{
Staged repair of tetralogy of Fallot and diminutive pulmonary arteries with a fenestrated ventricular septal defect patch
}

\author{
Audrey C. Marshall, MD \\ Barry A. Love, MD ${ }^{\text {a* }}$ \\ Peter Lang, $\mathrm{MD}^{\mathrm{a}}$ \\ Richard A. Jonas, $M D^{b}$ \\ Pedro J. del Nido, MD ${ }^{\mathrm{b}}$ \\ John E. Mayer, MD \\ James E. Lock, MD
}

From the Departments of Cardiology ${ }^{\mathrm{a}}$ and Cardiovascular Surgery, ${ }^{\mathrm{b}}$ Children's Hospital, Boston, Mass.

Received for publication Dec 11, 2002; revisions requested Feb 10, 2003; revisions received Feb 24, 2003; accepted for publication April 11, 2003.

Address for reprints: Audrey C. Marshall, MD, Department of Cardiology, Children's Hospital, 300 Longwood Ave, Boston, MA 02115 (E-mail: Audrey.marshall@cardio. chboston.org).

* Dr Love is currently affiliated with the Division of Pediatric Cardiology, Mt Sinai Medical Center, New York, NY.

J Thorac Cardiovasc Surg 2003;126: 1427-33

Copyright $\odot 2003$ by The American Association for Thoracic Surgery

$0022-5223 / 2003 \$ 30.00+0$

doi:10.1016/S0022-5223(03)01182-6
Objectives: Patients with tetralogy of Fallot and diminutive pulmonary arteries are at risk for suprasystemic right ventricular pressure and right ventricular failure after complete repair. We report the short-term outcome and medium-term follow-up after using a fenestrated ventricular septal defect patch as a component of staged repair in selected patients.

Methods: We reviewed 47 patients with tetralogy of Fallot and diminutive pulmonary arteries whose ventricular septal defect patch was fenestrated, either electively or as a rescue technique, at a single institution between 1984 and 2001.

Results: Early mortality was $10.6 \%$ and occurred only in patients who underwent rescue fenestration. Review of medium-term follow-up (median, 39 months) revealed 4 late deaths; an additional 4 patients experienced right ventricular failure despite fenestration. Most (7/8) of these late events occurred in patients who underwent planned fenestration. Excessive left-to-right shunt through the fenestration developed in only 2 patients.

Conclusions: Fenestrated patch closure of the ventricular septal defect in patients with tetralogy of Fallot and diminutive pulmonary arteries resulted in $10.6 \%$ early mortality. Used preemptively in selected patients, this technique is associated with no surgical mortality and a low incidence of excessive left-to-right shunt (4\%). Early survivors remain at risk for late death and right ventricular failure despite fenestration.

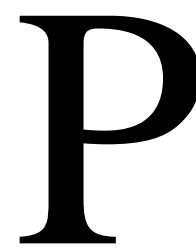

atients with tetralogy of Fallot (TOF) and pulmonary atresia who have absent or diminutive mediastinal pulmonary arteries (PAs) are at risk for right ventricular (RV) hypertension and poor outcome after complete repair. ${ }^{1-3}$ In the mid-1980s we began to uniformly use a strategy of staged repair for these patients. ${ }^{4}$ In addition to decreasing overall mortality, we hoped to recruit pulmonary vasculature, reduce rightto-left shunts, and preserve RV function. This staged approach prescribed early establishment of RV to PA continuity, catheter-based intervention to increase pulmonary conductance, and, ultimately, ventricular septal defect (VSD) closure. ${ }^{5,6}$ Inadequate relief of complex RV outflow obstruction before VSD closure risked suprasystemic RV pressure, with resultant RV failure.

We performed our first VSD patch fenestration in 1984 and continued to use fenestration as a rescue technique for patients with suprasystemic RV pressure coming off cardiopulmonary bypass (CPB) until 1991. This early experience sug- 


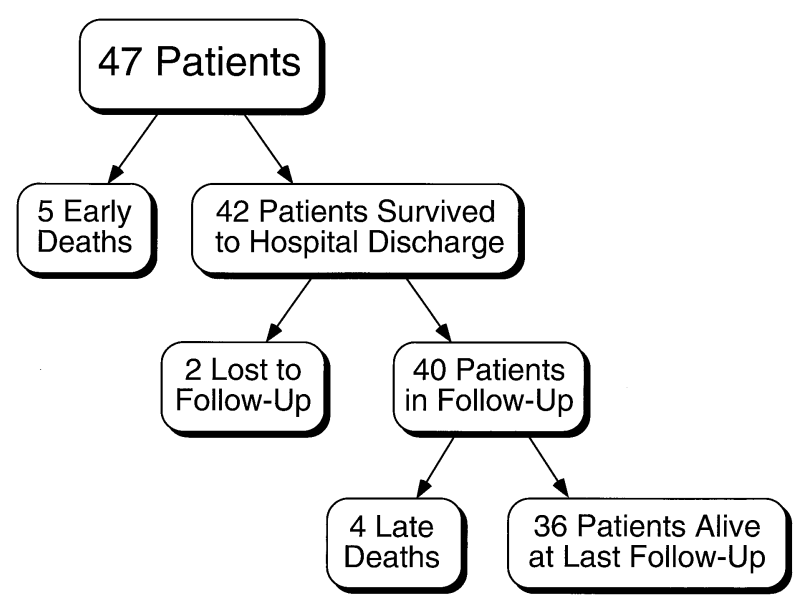

Figure 1. Flow diagram depicting dispositions of all 47 retrospectively identified patients with TOF who had fenestrated patch closure of VSD.

gested the subsequent use of planned (elective) fenestration in selected patients. We reasoned that a controlled residual VSD, limiting RV pressure at the expense of systemic desaturation, would reduce mortality in the postoperative period. Furthermore, the availability of transcatheter devices allowed for nonsurgical defect closure after further interventions to improve pulmonary runoff. ${ }^{7}$ In this study we describe early and late mortality associated with fenestrated patch VSD closure. We also report adverse outcomes in an attempt to identify patients with morbidity as a result of fenestration.

\section{Methods}

\section{Patient Identification}

We searched 4 databases to identify and obtain follow-up on patients with TOF and a fenestrated VSD patch: the computerized Cardiology Department database, the correspondence file from cardiologists and surgeons, and the regulatory databases maintained for the Clamshell and CardioSEAL high-risk trials.

\section{Anatomic and Clinical Data}

We examined cineangiograms and hemodynamic data obtained at catheterization for all patients. Pulmonary arterial size was determined according to the technique of Nakata and colleagues. ${ }^{2}$ If no mediastinal PAs could be identified, then no value was assigned. Ventricular pressures were drawn from the catheterization reports. In cases of pulmonary flow arising exclusively through an aortopulmonary shunt, PA oxygen saturations were measured directly or assumed to be equal to aortic saturation. In cases of dual sources of pulmonary flow, directly measured PA saturation values were assumed to represent mixed samples. Echocardiography reports were reviewed to determine RV pressure, functional status, and patency of the fenestration. RV pressures were estimated on the basis of Doppler velocity of the tricuspid regurgitant jet, and systolic ventricular function was assessed qualitatively. Review of operative reports yielded information on the size of the fenestra- tion, the technique used to create it, and the conditions under which it was placed (ie, planned or rescue).

Death, RV failure, and excessive left-to-right shunt comprised the adverse outcomes of interest. Heart-lung transplantation has not been used in the treatment of this disease at our center. Early death was defined as death occurring before hospital discharge. RV failure was defined as moderate-to-severe or severe RV dysfunction determined by means of echocardiography. We defined excessive left-to-right shunt as any shunt requiring intervention or a pulmonary/systemic flow ratio (Qp/Qs) of greater than 2:1 at catheterization. The fenestration was considered closed at the time of the first echocardiographic or angiographic examination unable to demonstrate transseptal flow.

Data are reported as a median with a range. After inspecting the preliminary data, we chose to divide the patients into 2 groups for the purposes of further analysis. Group 1 consisted of patients with planned fenestration, and group 2 included those whose fenestration constituted a rescue procedure. Comparisons between groups were performed by using the rank sum test and the Fisher exact test. Preoperative and postoperative variables were compared by using the signed-rank test for paired data. Freedom from death, RV failure, and fenestration closure were analyzed by using the Kaplan-Meier method. Permission for this retrospective chart review was obtained from the Committee on Clinical Investigation.

\section{Results}

Between 1984 and 2001, 47 patients (27 female and 20 male patients) with TOF and diminutive PAs underwent VSD closure with a fenestrated patch at our institution. Follow-up evaluations were performed at our institution in all but 2 patients, who had originally presented from outside the United Stages (Figure 1).

\section{Patient Characteristics}

The majority of patients (44/47 [94\%]) had TOF with pulmonary atresia, and 3 patients had TOF with severe pulmonary stenosis. Median age at surgical intervention was 4 years ( 1 month to 30 years); 34 patients had previously undergone palliative surgery, and 21 of these procedures included an aortopulmonary shunt. We pursued preoperative catheterization to evaluate and augment the pulmonary vascular bed in all patients. Catheter interventions included balloon dilation and stenting of the branch PAs $(n=31)$. Coil occlusion of aortopulmonary collaterals was performed in patients $(\mathrm{n}=25)$ who met previously described criteria on the basis of the premise that these vessels represent unreliable sources of pulmonary blood flow. ${ }^{4,8}$ The median systemic arterial oxygen saturation at the preoperative catheterization was $83 \%(71 \%-100 \%)$, with a corresponding hemoglobin concentration of $15.3 \mathrm{~g} / \mathrm{dL}$ (11.7-19.1 g/dL) and a Qp/Qs of 1.1 (0.4-2.5). Pulmonary angiography allowed for assignment of a Nakata index in 41 of 47 patients. This value ranged from 27 to $193 \mathrm{~mm}^{2} / \mathrm{m}^{2}$, with a median value of $99 \mathrm{~mm}^{2} / \mathrm{m}^{2}$. 
TABLE 1. Group characteristics

\begin{tabular}{lccc}
\hline & $\begin{array}{c}\text { Group 1 (planned) } \\
\text { (n = 27) }\end{array}$ & $\begin{array}{c}\text { Group 2 (rescue) } \\
\text { (n = 20) }\end{array}$ & $\boldsymbol{P}$ value \\
\hline Age (y) & 5.3 & 3.5 & .56 \\
Prior shunt & $13(48 \%)$ & $8(40 \%)$ & .77 \\
Prior outflow tract & $15(55 \%)$ & $6(30 \%)$ & .14 \\
Nakata index $\left(\mathrm{mm}^{2} / \mathrm{m}^{2}\right)$ & 99 & 96 & .59 \\
Prior catheter & $22(81 \%)$ & $9(45 \%)$ & .01 \\
$\quad$ intervention & & & \\
Unifocalized & $14(52 \%)$ & $6(30 \%)$ & .15 \\
Circulatory arrest & $4(15 \%)$ & $4(25 \%)$ & .47 \\
Fenestration size $(\mathrm{mm})$ & 4 & 5 & .10 \\
Early death & 0 & $5(25 \%)$ & .01 \\
Late death & $3(12 \%)$ & $1(5 \%)$ & .62 \\
RV failure & $4(16 \%)$ & $0(0 \%)$ & .12 \\
Excessive shunt & $2(8 \%)$ & $1(5 \%)$ & 1.00 \\
\hline
\end{tabular}

Values are presented as a median or as the number of patients within the group, with the percentage of patients in the group in parentheses.

\section{Operation}

At the time of operation, 41 patients underwent right ventricular outflow tract (RVOT) reconstruction before placement of the fenestrated VSD patch. This reconstruction consisted of establishment of right ventricle to PA continuity in 25 patients and revision of an existing outflow tract in an additional 16 patients. A pulmonary valve was incorporated in 36 of the RVOTs, which were predominantly homografts. A quarter of the patients $(n=12)$ had surgical enlargement of branch PAs before VSD closure. In 18 patients, some of whom had undergone previous unifocalization, pulmonary vessels were unifocalized at the time of repair. In total, 20 patients had unifocalization before or at the time of repair.

Fenestrations were created either by excising a central portion of the patch or by using a punch device (most commonly $4 \mathrm{~mm}$ ). The defects were roughly circular, at least $2 \mathrm{~mm}$ in diameter, and centrally located in the patch to facilitate future transcatheter closure. In 27 patients $(57 \%$; group 1, planned) Dacron patches were electively fenestrated at the time of VSD closure. In the other 20 patients (43\%; group 2, rescue), fenestrations were placed in the VSD patch after an initial weaning from $\mathrm{CPB}$ resulted in suprasystemic RV pressure or evidence of acute RV failure. In 4 of these cases the patients were initially taken to the intensive care unit postoperatively with an intact patch and later returned to the operating room for fenestration. The remaining 16 patients who underwent rescue fenestration did so immediately, although 2 required later fenestration enlargement.

\section{Analysis by Group}

The 2 groups were similar with regard to a number of preoperative and operative variables (Table 1). Because of our increasing use of the planned patch fenestration, patients

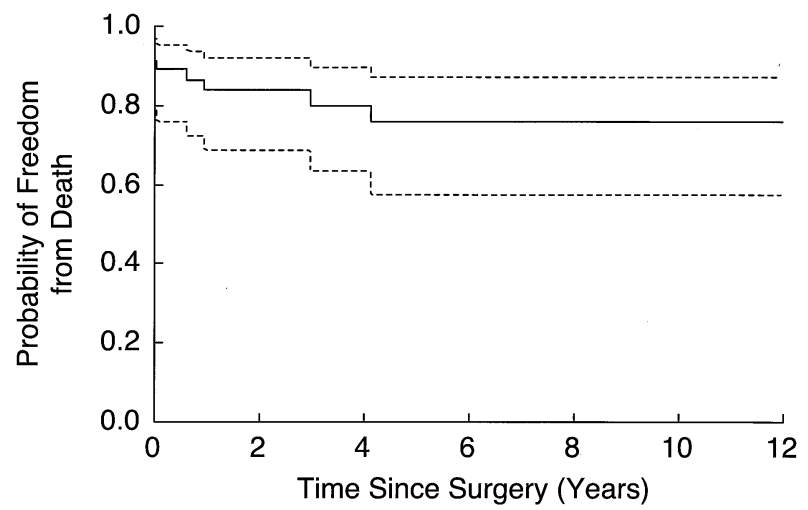

Figure 2. Actuarial survival after fenestrated patch closure of VSD in 47 patients with TOF and diminutive PAs. Dotted lines represent $95 \%$ confidence limits.

in group 2 were more likely to have been operated on before $1995(16 / 20$ [80\%] vs 7/27 [26\%], $P<.001)$. Group 1 patients were somewhat more likely to have a preexisting RVOT and were more likely to have had catheter intervention to improve the pulmonary vascular bed before repair $(P$ $=.01$ ).

There were 5 early deaths, occurring within 6 days of the operation. Early deaths occurred only in group 2, the rescue group. These deaths were attributable to suprasystemic RV pressure, acute RV failure, and low cardiac output (Table 2). Both patients who required fenestration enlargement died. Patients who died were younger than survivors ( 0.9 vs 5.0 years, $P=.04$ ), with a nonsignificant tendency toward a lower Nakata index than survivors (median $=67$ vs 100 $\left.\mathrm{mm}^{2} / \mathrm{m}^{2}, P=.18\right)$.

Two patients were supported postoperatively with extracorporeal membrane oxygenation (ECMO). One of these was among the early deaths. The second patient was supported for 48 hours, was successfully decannulated, and survived to hospital discharge 14 days later. Two patients received inhaled nitric oxide in an attempt to attenuate postoperative pulmonary hypertension. None of the patients required VSD closure before hospital discharge.

\section{Follow-up}

Median duration of follow-up was 39 months (range, 1-146 months). Postdischarge evaluations, available for 40 of 42 early survivors, included echocardiography and catheterization. In 29 patients with adequate echocardiographic studies to estimate RV pressure, the ratio of RV to systemic arterial pressure was 0.72 (0.35-1.07). At postoperative catheterization, directly measured RV pressures were $89 \%(60 \%$ $110 \%$ ) of systemic pressure in 39 patients. Patients with RV pressures at or above systemic levels had further catheter interventions to improve pulmonary runoff. Late fenestration enlargement or recreation of the fenestration was not 
TABLE 2. Early deaths

\begin{tabular}{llll}
\hline Age & Nakata index & Year & Description \\
\hline $3 \mathrm{y}$ & $67 \mathrm{~mm}^{2} / \mathrm{m}^{2}$ & 1984 & $\begin{array}{c}\text { Suprasystemic in ICU. Returned to OR next day to create fenestration. } \\
\text { Died in OR. } \\
\text { fuprasystemic in ICU. Returned to OR on POD } 3 \text { to enlarge } \\
\text { fenestration. Died on arrival to ICU. }\end{array}$ \\
$5 \mathrm{~d}$ & $53 \mathrm{~mm}^{2} / \mathrm{m}^{2}$ & 1985 & $\begin{array}{c}\text { ECMO for low cardiac output from OR until reoperation on POD 6. } \\
\text { Died in OR. }\end{array}$ \\
$3 \mathrm{mo}$ & $108 \mathrm{~mm}^{2} / \mathrm{m}^{2}$ & 1986 & $\begin{array}{c}\text { Suprasystemic in ICU. Returned to OR same day to create } \\
\text { fenestration. Died on POD 1. }\end{array}$ \\
$4 \mathrm{y}$ & $99 \mathrm{~mm}^{2} / \mathrm{m}^{2}$ & 1990 & $\begin{array}{c}\text { Suprasystemic in OR. Fenestration enlarged. Persistent low output } \\
\text { and cardiac arrest. Died on POD 1. }\end{array}$
\end{tabular}

Early death was defined as death occurring before hospital discharge. $I C U$, Intensive care unit; $O R$, operating room; $P O D$, postoperative day.

attempted in any of the patients. Arterial oxygen saturations were significantly higher than preoperative values $(92 \%$ vs $83 \%, P<.001)$, and hemoglobin levels were lower (13.5 vs $15.3 \mathrm{~g} / \mathrm{dL}, P<.009)$. The $\mathrm{Qp} / \mathrm{Qs}$ ratio remained unchanged for the group as a whole (1.1 preoperatively vs 1.0 postoperatively).

Four patients died between 8 months and 4 years postoperatively, resulting in $76 \%$ actuarial survival at 5 years (confidence interval $[\mathrm{CI}]=57 \%-87 \%$, Figure 2 ). Three of the 4 late deaths occurred in patients in the planned fenestration group. Patients with late mortality were not younger than the remainder of the follow-up group $(P=.50)$ and did not have significantly different Nakata indexes $(P=.80)$. The causes of death varied. The first patient died in the hospital 8 months postoperatively, when he presented with an acute, infectious pulmonary process. The second patient died in the hospital of severe, idiopathic left ventricular (LV) dysfunction, presenting 3 years after the operation. The third patient died at catheterization 4 years postoperatively as a result of uncontrolled hemorrhage after a complicated PA dilation. This patient and his complication have been reported previously. ${ }^{9}$ The final patient who experienced late death underwent a rescue fenestration and died suddenly at home 1 year after the operation. After his repair, he had undergone successful surgical intervention for closure of an LV to right atrial shunt. He also had a history of atrial and ventricular arrhythmias for which he was being medically treated.

Four patients had RV failure evidenced by qualitatively severely decreased RV function and associated tricuspid regurgitation. All had strikingly poor pulmonary vasculature. Two patients did not have measurable Nakata indexes, and the other 2 patients had indexes of 36 and $65 \mathrm{~mm}^{2} / \mathrm{m}^{2}$. All went to the operating room anticipating marginal hemodynamics and fenestration of the VSD patch, and one of the 4 required ECMO support for 48 hours. RV failure, diagnosed between 6 and 24 months postoperatively, developed despite a patent fenestration in all 4 patients.
Both death and RV failure occurred years after fenestrated VSD closure but were rare among early survivors of rescue fenestration. Only one of 15 early survivors in group 2 died during follow-up, and none had late RV failure. In contrast, 7 of 27 early survivors in group 1 went on to experience death or RV failure in the follow-up period. As a whole, $66 \%$ of patients remained free from death or RV failure at 5 years after the operation $(\mathrm{CI}=48 \%-79 \%)$.

Excessive left-to-right shunt developed in 3 patients; all other survivors had a $\mathrm{Qp} / \mathrm{Qs}$ of less than 1.5:1. In one patient persistence of a large shunt after transcatheter closure of a residual VSD led to the diagnosis of an unrecognized LV to right atrial defect. This lesion was closed operatively, but the patient died suddenly months later, as was discussed earlier. Two additional patients required VSD closure. The first of these patients had required rescue fenestration, despite a Nakata index of $167 \mathrm{~mm}^{2} / \mathrm{m}^{2}$. She had a Qp/Qs of 1.7:1 at postoperative catheterization and had her VSD closed surgically. The second patient, with a Nakata index of $115 \mathrm{~mm}^{2} / \mathrm{m}^{2}$, underwent planned fenestration and proceeded to have PA dilations at 3 catheterizations over the next 5 years. At the conclusion of the third procedure, his Qp/Qs was 3:1, and a device was placed in his residual VSD. Thus only one patient who was electively fenestrated $(1 / 27[4 \%])$ eventually required closure of the defect because of excessive left-to-right shunting.

By 1 year after the operation, 39\% (CI $=24 \%-57 \%)$ of the fenestrations were closed (Figure 3). Among patients returning for follow-up, 24 (24/40 [60\%]) were known to have a closed fenestration at their most recent examination. Nearly half of these fenestrations closed spontaneously $(11 / 24$ [46\%]). Other mechanisms of closure included transcatheter device placement (7/24 [29\%]) and surgical patching $(6 / 24[25 \%])$. The intended benefits of interventional closure included reduction of LV volume load and elimination of the potential for cyanosis or paradoxical emboli. In 5 of 6 patients undergoing surgical defect closure, this was accomplished at the same operation as additional RVOT 
augmentation. Overall, 26\% (11/42) of the early survivors required a subsequent operation for purposes of RVOT augmentation. Patients who did not require reoperation were considered for catheter closure of the fenestration at the time of additional PA interventions (Figure 4).

\section{Discussion}

Despite a number of proposed methods to predict which patients with TOF and diminutive PAs will tolerate complete repair, none has proved to be ideal. ${ }^{1,2,10,11}$ Early 1-stage unifocalization, when performed in conjunction with an intraoperative pulmonary flow study, has allowed complete repair in $66 \%$ of patients, with a $10.6 \%$ perioperative mortality. ${ }^{12}$ Staged repair, although requiring multiple operations and deferring relief of right-to-left shunt, allows for VSD closure to be considered over time in the context of ongoing RVOT and PA optimization. A recent report demonstrated the benefits of staged repair in a large series of patients with TOF and pulmonary atresia. ${ }^{13}$ The authors found that the need to reopen a VSD in the operating room was predictive of both early and late mortality in patients undergoing complete repair.

In 1988, Kirklin and colleagues ${ }^{3}$ reported risk factors for death after complete repair of TOF/PA, encompassing an experience of nearly 20 years. During this time period, the authors found 17 patients in whom complete repair failed, with an RV/LV pressure ratio of greater than 1:1. In these patients the VSD patch was perforated or removed. Outcomes after partial or complete takedown of the VSD patch were not discussed in the body of the article, but the authors noted that $8(47 \%)$ of these 17 patients died in the hospital, and 2 patients died later. In a series of 34 patients with pulmonary atresia, VSD, and multiple aortopulmonary collaterals reported by Sawatari and associates, ${ }^{14} 4$ had suprasystemic RV pressure after VSD closure. The authors described perforation of the VSD patch in these 4 patients, with one late death. Iyer and Mee ${ }^{15}$ reported a series of 58 patients, in which 2 of 3 late deaths occurred in patients who had suprasystemic RV pressures and returned to the operating room for patch perforation.

We reviewed our own experience with fenestrated patch VSD closure in a selected group of patients with TOF and diminutive PAs. Within this retrospectively identified group of patients with a median Nakata index of $99 \mathrm{~mm}^{2} / \mathrm{m}^{2}$, PA anatomy and prior surgical interventions were highly varied. These patients had catheterization and operations performed at our center over a 17-year time period, during which we attempted to standardize our approach to this heterogeneous population. Initially, we used patch fenestration as a rescue procedure for those patients who failed to be separated from CPB after complete repair. We ultimately used the fenestrated VSD patch in an elective, anticipatory manner.

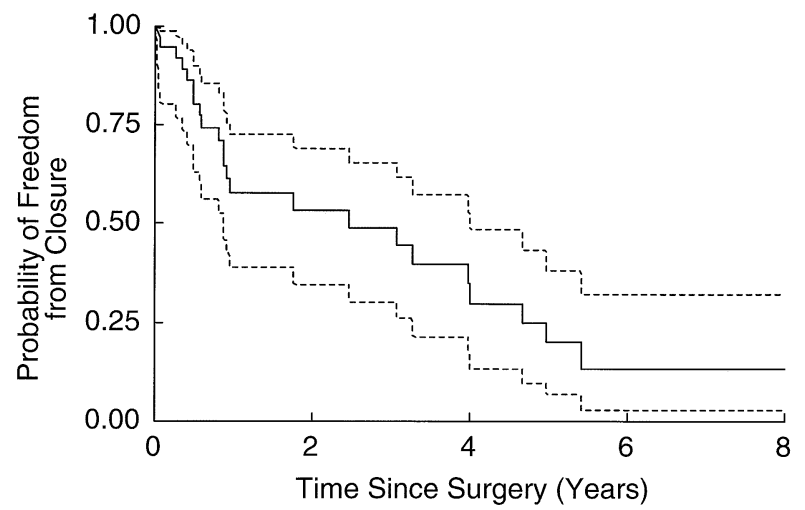

Figure 3. Freedom from fenestration closure. Dotted lines represent $95 \%$ confidence limits.

In our series of 47 selected patients, early mortality was $10.6 \%$. Early death occurred only in patients who had fenestrations placed as a rescue maneuver after initially failing to be separated from CPB. There were no early deaths among the 27 patients who received elective patch fenestration.

Given that most of the electively fenestrated patients were operated on in the latter half of the described experience, a number of improvements in the overall care of these patients are likely to have contributed to this clear difference in postrepair mortality. Patients in group 1 were somewhat more likely to have established right ventricle to PA continuity before repair and were more likely to have undergone interventional catheterization for pulmonary arterial rehabilitation. Improvements in surgical RVOT reconstruction or PA plasty might also have contributed to lower early mortality, and subtle changes in surgical technique were not investigated in this study. Need for circulatory arrest did not differ between the 2 groups. Despite the increasing availability of inhaled nitric oxide and ECMO during the study period, these modalities were used infrequently in this population of patients.

We propose that the incidence of early death in this group of patients was minimized by placing a fenestration at the time of VSD closure and protecting the postoperative right ventricle from suprasystemic pressure. Furthermore, placing the fenestration in an anticipatory way appears to have been more protective than placing it as a rescue maneuver. Patients in group 2 might have been more vulnerable because of the brief exposure to suprasystemic RV pressure before fenestration, as well as the need for reinstitution of CPB after the determination of RV failure.

In follow-up none of the survivors in group 2 had late $\mathrm{RV}$ failure, and the group experienced only one late death. This might be due to selection imposed during the immediate postoperative period. The patients in group 2 with the poorest pulmonary runoff and the highest risk of acute RV 

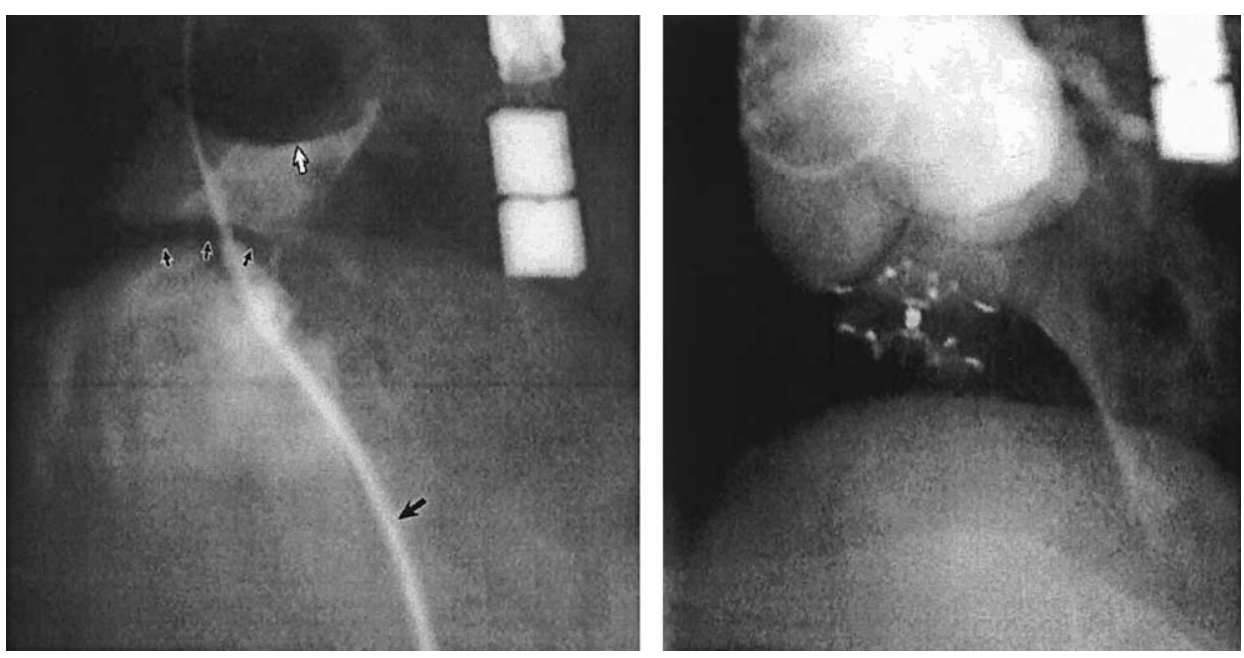

Figure 4. Angiograms taken during device closure of a patch fenestration. Left, An angiographic catheter passes from the right ventricle to the left ventricle through the fenestration in the ventricular septal patch. The large black arrow identifies the catheter in the right ventricle. Small black arrows demonstrate curvature of the VSD patch. The large white arrow points out the underside of the aortic cusp. Right, After placement of a double-umbrella device, angiography at the level of the aortic valve outlines the LV surface of the septum. The central body of the device sits in the fenestration, with 4 arms on either side of the septum.

failure might have succumbed postoperatively, whereas patients in group 1 did not die early but manifested their persistent pulmonary vascular inadequacy by having RV failure in follow-up.

Among the 27 early survivors in group 1, there were 3 late deaths. In addition, 4 patients had RV failure. The risk of RV failure presumably persisted on the basis of a severely impaired pulmonary vascular bed with distal abnormalities and microvascular changes. ${ }^{16}$ Fenestration might have permitted survival through the postoperative period, but underlying pulmonary anatomy continued to impose a chronic stress on the right ventricle. Although potentially beneficial, fenestration enlargement has not been pursued in these patients in favor of additional interventions to minimize RVOT obstruction and to maximize pulmonary runoff.

Of 3 patients who required intervention for excessive left-to-right shunt, 2 were in the rescue group and had already demonstrated their inability to support a postoperative circulation in the absence of a fenestration. The mechanism of residual shunt in one of these patients was left ventricle to right atrium, and despite surgical correction, this patient died in follow-up. Puga and colleagues ${ }^{17}$ have previously reported severe RV failure as the result of an LV to right atrial shunt after repair. The single patient in our series who had an elective fenestration and subsequently required VSD closure did so only after multiple PA dilations led to excessive pulmonary flow.

Follow-up has shown that many of these fenestrations with a median size of $4 \mathrm{~mm}$ will close spontaneously. In the event of persistence of the fenestration, the size and location of the residual defect make it amenable to either surgical or transcatheter closure. Reoperation is often necessary for revision of the RVOT in these patients, at which time the fenestration can be closed. ${ }^{18}$ Similarly, these patients undergo additional catheterizations for ongoing management of PA stenoses and hypoplasia. ${ }^{12,19,20}$ At these procedures, fenestrations can be closed by using a catheter-based device. $^{7,21}$

Our adoption of an institutional approach to management of this disease limits our ability to generalize our findings, and the small number of patients involved precludes any detailed analysis of predictors. However, our results after planned fenestration are in distinct contrast to the figures presented by Kirklin and colleagues ${ }^{3}$ and Cho and coworkers ${ }^{13}$ which depict a high mortality associated with a need for reopening the VSD, even partially. More recent techniques, such as early 1-stage unifocalization and repair, have also yielded improved outcomes over past experience. At this time, all of these approaches remain vulnerable to inappropriate decisions regarding VSD closure. Regardless of the primary management strategy, elective patch fenestration could minimize adverse outcomes after repair in the setting of an inadequate pulmonary vascular bed. We currently place an elective fenestration in the VSD patch in the absence of a net left-to-right shunt preoperatively, with limited opportunity to improve PA runoff at the time of the operation. Through additional clinical experience, we plan to improve patient selection and fenestration techniques.

In the absence of methods to reliably predict readiness for complete repair, routine application of a modified, 
staged, surgical approach has allowed us to improve the survival of patients with TOF and diminutive PAs. This modified approach uses a fenestration in the ventricular septal patch. The fenestration can be placed after VSD closure to rescue patients who are unable to be separated from CPB. However, early survival is greatest when the fenestration is placed in an elective manner. Late mortality and RV failure occur in a small percentage of patients despite use of this technique and might be attributable to inadequate preparation of the pulmonary vascular bed. Risk of excessive left-to-right shunt postoperatively is low when the fenestration is limited to approximately $4 \mathrm{~mm}$, and many of the fenestrations will close spontaneously or can be closed at subsequent surgical or catheterization procedures.

We thank Kimberlee Gauvreau for her invaluable assistance with the statistical analysis, and Emily and Bill McIntosh for their assistance with the preparation of the figures.

\section{References}

1. Kirklin JW, Blackstone EH, Kirklin JK, Pacifico AD, Aramendi J, Bargeron LM Jr. Surgical results and protocols in the spectrum of tetralogy of Fallot. Ann Surg. 1983;198:251-65.

2. Nakata S, Imai Y, Takanashi Y, Kurosawa H, Tezuka K, Nakazawa M, et al. A new method for the quantitative standardization of crosssectional areas of the pulmonary arteries in congenital heart diseases with decreased pulmonary blood flow. $J$ Thorac Cardiovasc Surg. 1984;88:610-9.

3. Kirklin JW, Blackstone EH, Shimazaki Y, Maehara T, Pacifico AD, Kirklin JK, et al. Survival, functional status, and reoperations after repair of tetralogy of Fallot with pulmonary atresia. J Thorac Cardiovasc Surg. 1988;96:102-16.

4. Castaneda AR, Mayer JE, Lock JE. Tetralogy of Fallot, pulmonary atresia, and diminutive pulmonary arteries. Prog Pediatr Cardiol. 1992;1:50-60.

5. Rome JJ, Mayer JE, Castaneda AR, Lock JE. Tetralogy of Fallot with pulmonary atresia: rehabilitation of the pulmonary arteries. Circulation. 1993;88:1691-8.

6. Kreutzer J, Perry SB, Jonas RA, Mayer JE, Castaneda AR, Lock JE. Tetralogy of Fallot with diminutive pulmonary arteries: preoperative pulmonary valve dilation and transcatheter rehabilitation of pulmonary arteries. J Am Coll Cardiol. 1996;27:1741-7.

7. Lock JE, Block PC, McKay RG, Baim DS, Keane JF. Transcatheter closure of ventricular septal defects. Circulation. 1988;78:361-8.

8. Perry SB, Radtke W, Fellows KE, Keane JF, Lock JE. Coil emboli- zation to occlude aortopulmonary collateral vessels and shunts in pts with congenital heart disease. J Am Coll Cardiol. 1989;13:100-8.

9. Baker CM, McGowan FX, Keane JF, Lock JE. Pulmonary artery trauma due to balloon dilation: recognition, avoidance and management. J Am Coll Cardiol. 2000;36:1684-90.

10. McGoon DC, Baird DK, Davis GD. Surgical management of large bronchial collateral arteries with pulmonary stenosis or atresia. Circulation. 1975;52:109-18.

11. Reddy VM, Petrossian E, McElhinney DB, Moore P, Teitel DF, Hanley FL. One-stage complete unifocalization in infants: when should the ventricular septal defect be closed? J Thorac Cardiovasc Surg. 1997;113:858-68.

12. Reddy VM, McElhinney DB, Amin Z, Moore P, Parry AJ, Teitel DF, et al. Early and intermediate outcomes after repair of pulmonary atresia with ventricular septal defect and major aortopulmonary collateral arteries: experience with 85 patients. Circulation. 2000;101: 1826-32.

13. Cho JM, Puga FJ, Danielson GK, Dearani JA, Mair DD, Hagler DJ, et al. Early and long-term results of the surgical treatment of tetralogy of Fallot with pulmonary atresia, with or without major aortopulmonary collateral arteries. J Thorac Cardiovasc Surg. 2002;124:70-81.

14. Sawatari K, Imai Y, Kurosawa H, Isomatsu Y, Momma K. Staged operation for pulmonary atresia and ventricular septal defect with major aortopulmonary collateral arteries. J Thorac Cardiovasc Surg. 1989;98:738-50.

15. Iyer KS, Mee RB. Staged repair of pulmonary atresia with ventricular septal defect and major systemic to pulmonary artery collaterals. Ann Thorac Surg. 1991;51:65-72.

16. Rabinovitch M, Herrera-deLeon V, Castaneda AR, Reid L. Growth and development of the pulmonary vascular bed in patients with tetralogy of Fallot with or without pulmonary atresia. Circulation. 1981;64:1234-49.

17. Puga FJ, Leoni FE, Julsrud PR, Mair DD. Complete repair of pulmonary atresia, ventricular septal defect, and severe peripheral arborization abnormalities of the central pulmonary arteries: experience with preliminary unifocalization procedures in 38 patients. J Thorac Cardiovasc Surg. 1989;98:1018-29.

18. Pigula FA, Khalil PN, Mayer JE, del Nido PJ, Jonas RA. Repair of tetralogy of Fallot in neonates and young infants. Circulation. 1999; 100(suppl II):157-61.

19. Gentles TL, Lock JE, Perry SB. High pressure balloon angioplasty for branch pulmonary artery stenosis: early experience. J Am Coll Cardiol. 1993;22:867-72.

20. Pagani FD, Cheatham JP, Beekman RH, Lloyd TR, Mosca RS, Bove EL. The management of Tetralogy of Fallot with pulmonary atresia and diminutive pulmonary arteries. J Thorac Cardiovasc Surg. 1995; 110:1521-33.

21. Rocchini A, Lock JE. Defect closure: umbrella devices. In: Lock JE, Keane JF, Perry SB, editors. Diagnostic and interventional catheterization in congenital heart disease. 2nd ed. Boston: Kluwer Academic Publishers; 2000. p. 179-98. 